\title{
MAYO CLINIC \\ Segmentation of Brain Anatomy in Head CTs for Early Detection of Ischemic Stroke Using Deep Learning
}

(Di)

Zeynettin Akkus, PhD, Jason Cai, MD, Arrunit Boonrod, MD, Kenneth A. Phillbrick, PhD, Bradley J. Erickson, MD, PhD Radiology Informatics Lab, Department of Radiology

Mayo Clinic, Rochester, MN

\section{Background}

There is a significant link between heart disease and stroke, and both are leading causes of morbidity and mortality in developed countries. Furthermore, as effective treatments for

hypertension and dyslipidemia become available cardioembolic stroke now accounts for an increasing proportion of ischemic stroke subtypes and it is a major complication of heart disease (see Figure 1). In this study, we propose the adoption of deep learning to segment normal brain anatomy in head CTs. This will subsequently be used to develop an algorithm for early detection of ischemic stroke.

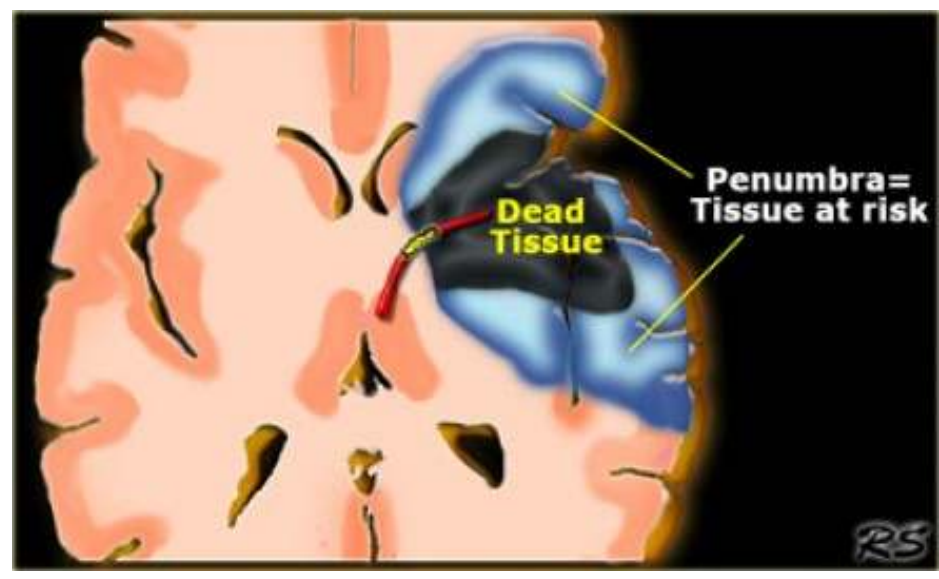

Figure 1: An illustration of ischemic Stroke that shows dead tissue and tissue at risk.

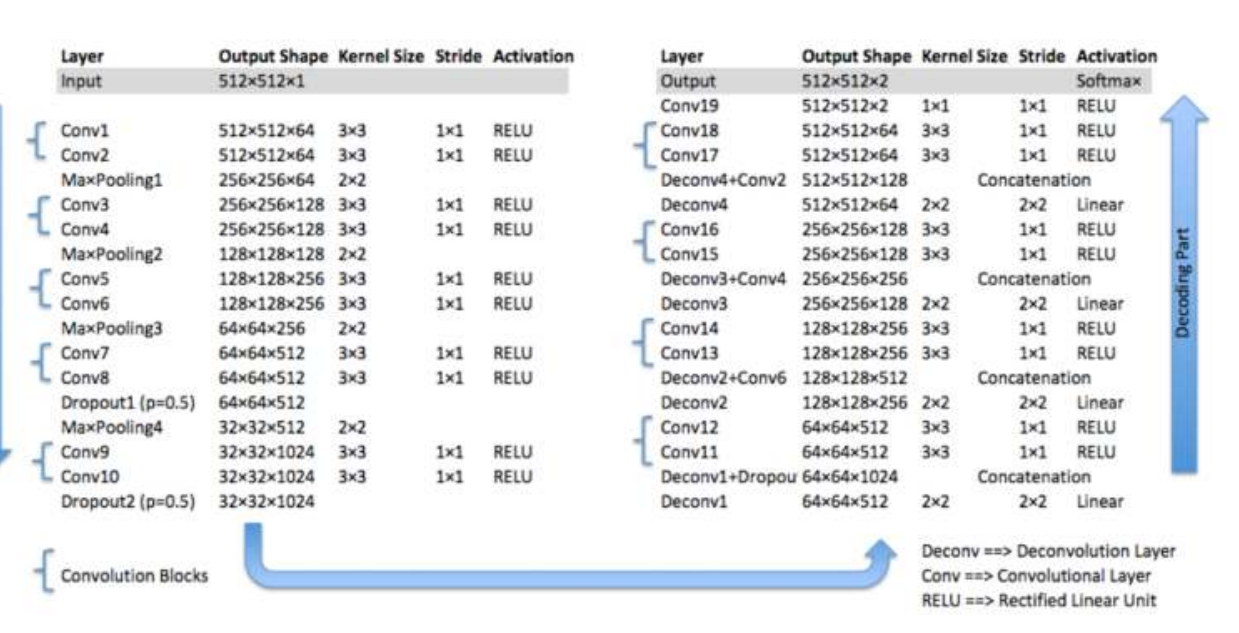

Figure 2: The parameters used for Unet CNN artchitecture [1]. Decon+Conv shows the concatenation of layers from the encoding and decoding parts, also called skip connections.

References

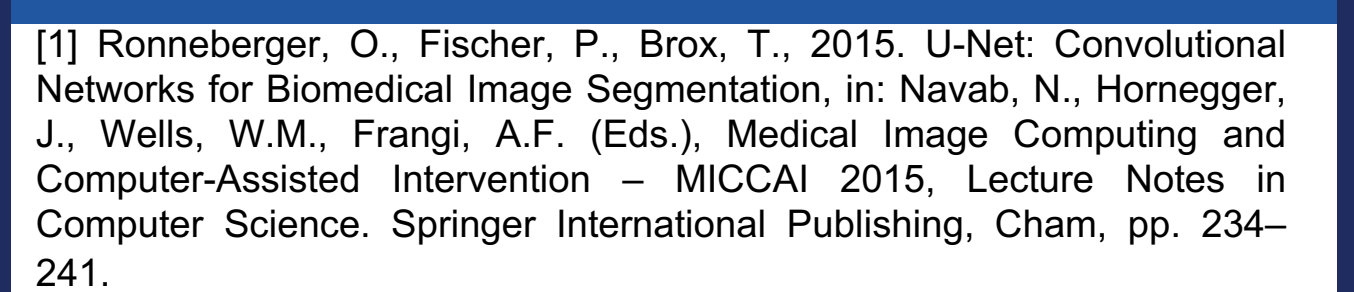

Proposed Method

Thirty-five non-contrast head CTs with no significant intracranial abnormalities except brain parenchymal atrophy and leukokariosis were collected from the Mayo research PACS. Patient age ranged from 27 to 90 years old. These scans (consisting of 1401 individual slices) were manually segmented into 12 classes by a team of trained observers lead by an experienced neuroradiologist. A two-dimensional $\mathrm{CNN}$ based on the U-Net architecture (see Figure 2) was trained on 1815 axial slices (42 volumes). Data augmentation was employed to reduce overfitting. Due to the inherently unbalanced distribution of classes in our dataset, we applied a voxel-based weighting strategy during the training process. We also ensured equal representation of all classes by dividing the brain into three regions at, above and below the basal ganglia level; during each batch gradient descent operation, we selected a random slice from each of these three regions to perform a single weight update. Performance of the CNN was then evaluated on 164 slices (4 volumes) using dice scores. To assess the interobserver variability, 25 slices of 5 volumes were segmented by two experts and their results were compared to the automated CNN results (see Figure 3 ).

Results (Video)
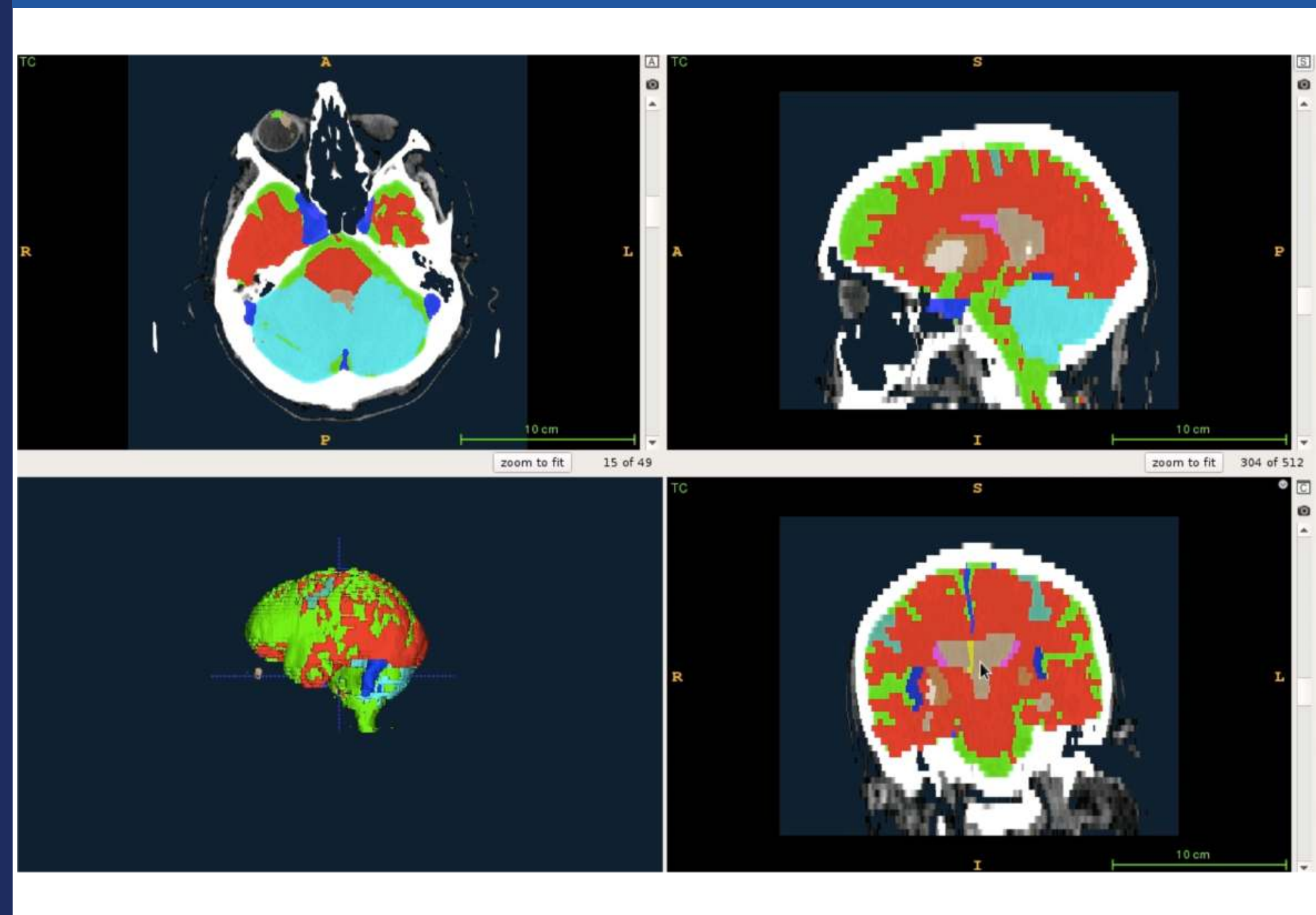

\section{Results}

The dice score of our model when evaluated against manually generated labels are as follows: Brain: 0.94; caudate nucleus: 0.79 ; lentiform nucleus: 0.81 ; insular cortex: 0.70; internal capsule: 0.67; cerebellum: 0.89; CSF: 0.82 ; meninges and venous sinuses: 0.70 and septum pellucidum: 0.66 , ventricle: 0.84 , central sulcus: 0.49

Figure 3: Performance Evaluation on the Test Dataset

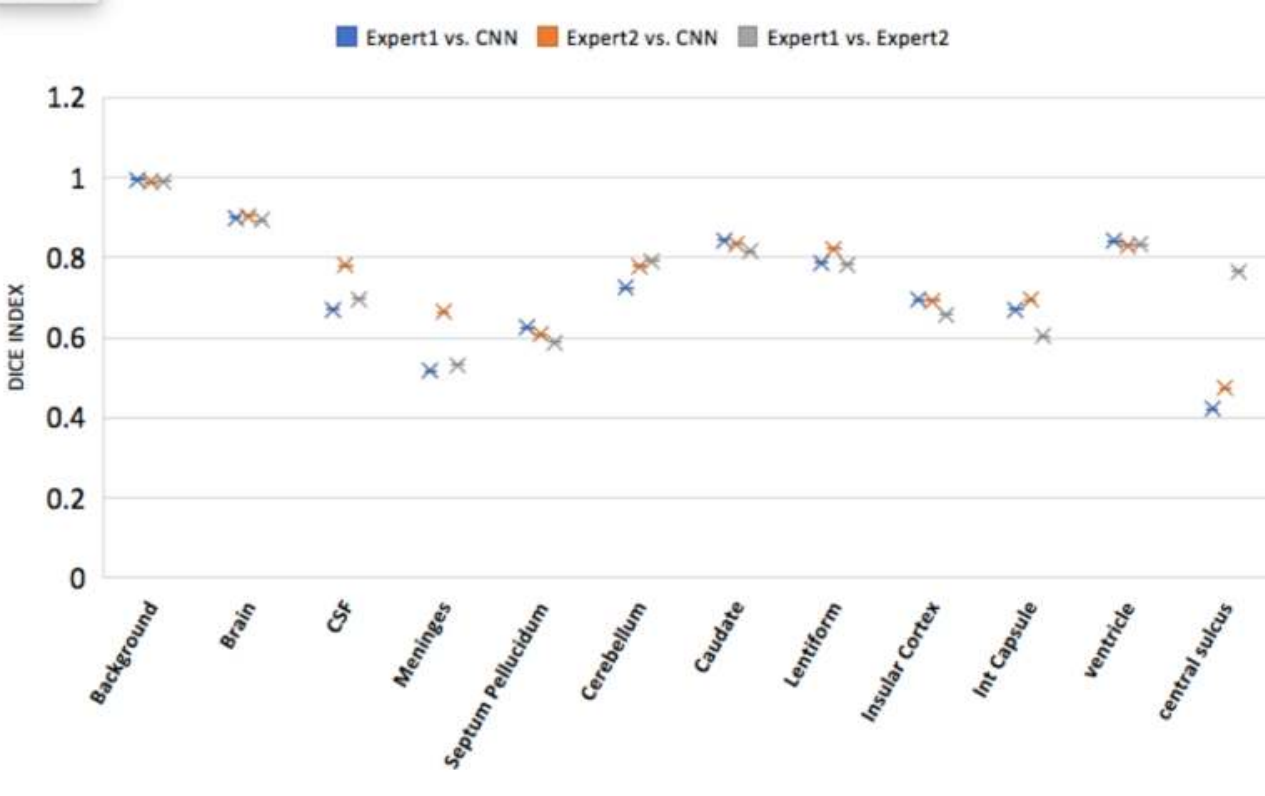

Discussion \& Conclusion

Semantic segmentation of head CTs is a challenging task because of the inherently poorer image quality as compared to MRI. This is made more difficult by unbalanced class distributions, which in itself is a problem that has been an active area of research in the deep learning community.

* We demonstrate that CNNs are capable of delivering good results on multi-class CT brain segmentation, and it does not require any feature engineering or additional user input.

- We anticipate further improvements on the performance and robustness of our model as we include more training data.

- Our work paves way for the development of an algorithm to detect early ischemic stroke, a leading cause of cardiovascular disease burden in developed countries. 